\title{
On the Information Content of Hyperspectral Infrared Observations with Respect to Mineral Dust
}

\author{
Lars Klüser \\ German Aerospace Center (DLR), Oberpfaffenhofen, Muenchener Strasse 20, 82234 Wessling, Germany \\ lars.klueser@dlr.de
}

\begin{abstract}
Desert dust is characterized by strong silicate absorption bands located within the atmospheric window region in the terrestrial infrared (TIR) between $8 \mu \mathrm{m}$ and $12 \mu \mathrm{m}$. These absorption bands and the corresponding optical properties (extinction efficiency, single scattering albedo, scattering phase function) have very specific spectral shapes for different silicate minerals, modulated by the particle size and shape. The asphericity of desert dust particles strongly affects the absorption band characteristics, for example due to surface wave modes for small particles. The use of the correct particle shape model significantly increases the spectral correlation between simulated dust optical properties for typical minerals and corresponding laboratory measurements for single minerals as well as for bulk dust from desert samples. The presence of absorption peaks and the spectral shape of the extinction signal carry dust information, which can be exploited for remote sensing purposes. With hyperspectral infrared methods it is thus possible to infer information beyond dust optical depth, that is to acquire information about dust particle size, composition and also vertical information. Examples of such information are shown for the Infrared Mineral Aerosol Retrieval Scheme (IMARS) which has been developed for the Infrared Atmospheric Sounding Interferometer (IASI) on board the European Metop satellite series. Another strong advantage of the hyperspectral signal from satellite instruments is the capability to minimize the influence of disturbing gas absorption lines within these bands. The probabilistic IMARS approach also directly provides the number of independent signals (variables) for each observation. For desert dust this number typically ranges from 2.5 to 4.0 depending on the characteristics of the observed dust plume. Consequently a lot more information beyond Aerosol Optical Depth (AOD) can be retrieved from these measurements.

OCIS codes: (010.0010) Atmospheric and oceanic optics; (010.1110) Aerosols
\end{abstract}

\section{Simulation of optical properties: The Asymptotic Approximation Approach}

Desert dust is characterized by strong silicate absorption bands located within the atmospheric window region in the terrestrial infrared (TIR) between $8 \mu \mathrm{m}$ and $12 \mu \mathrm{m}$. The corresponding optical property spectra of extinction efficiency, single scattering albedo, scattering phase function significantly depend on the mineralogy, the particle size distribution and the particle shape. Simulations of the optical properties on non-spherical dust particles with the Asymptotic Approximation Approach (AAA), which combines Rayleigh-limit approximations with geometric optics solutions for large particles, have been compared with laboratory measurements in order to select the best suited particle shape for a set of typically abundant desert dust minerals [3]. It has been found that clay minerals are best represented by disc-like particle shapes whereas other silicates such as quartz can be simulating by a continuous distribution of ellipsoids, representing the abundance of particles with various shapes.

Fig. 1 shows the comparison of AAA extinction efficiency spectra and Lorenz-Mie spectra with laboratory measurements of quartz and illite performed at the University of Iowa. It can easily be seen that the assumed particle shape has a large influence on the $Q_{\text {ext }}$ spectrum and that the simulations with non-spherical particles correlate much better with the measurements than the Lorenz-Mie simulations. As also single scattering albedo and scattering phase function are strongly affected by particle shape and the position of the extinction peaks and as these also vary with particle size, hyperspectral infrared spectra carry a lot of information about the physico-chemical properties of desert dust. 

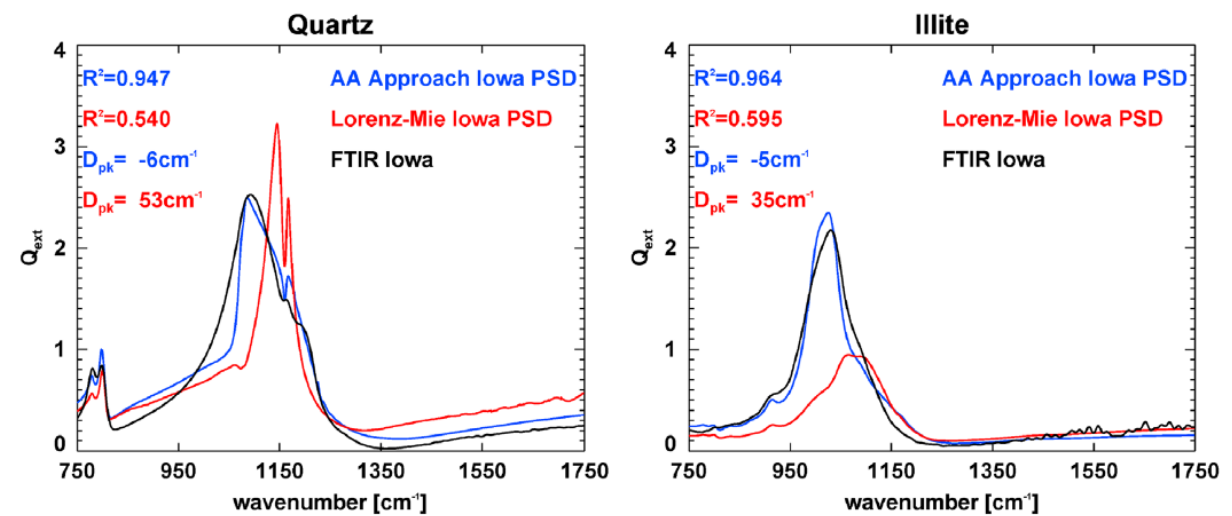

Fig. 1: Comparison of extinction efficiency spectra measured in the laboratory (black) with simulations by the AAA method (blue) and by Lorenz-Mie (red) for quartz (left) and illite (right). Laboratory measurements have been performed by the University of Iowa.

This information can be exploited for remote sensing purposes, acquiring dust information beyond aerosol optical depth (AOD), such as dust mineralogical composition, particle size and dust layer height. Moreover, the aforementioned dependence of the extinction spectra to particle asphericity can be exploited to infer an asphericity parameter as well.

\section{Dust observation from satellite: The IMARS retrieval}

The Infrared Mineral Aerosol Retrieval Scheme (IMARS) has been developed for the Infrared Atmospheric Sounding Interferometer (IASI) on board the European Metop satellite series [1-3]. IASI is a Michaelson interferometer with a spectral resolution of $0.5 \mathrm{~cm}^{-1}$ at a footprint size of $12 \mathrm{~km}$ at nadir onboard a polar orbiting satellite platform. IMARS exploits this hyperspectral signal for inferring dust information with the capability to minimize the influence of disturbing gas absorption lines within the observation bands.

The IMARS method is based on a probabilistic approach, which generates probability density functions (PDFs) of dust physico-chemical properties. Using approaches of classical information theory it also directly provides the number of independent signals (variables) as well as retrieval uncertainty for each observation. For desert dust the number of independent signal typically ranges from 2.5 to 4.0 depending on the characteristics of the observed dust plume. Consequently a lot more information beyond Aerosol Optical Depth (AOD) can be retrieved from these measurements. The method also combines dust and cloud retrieval and uses the a posteriori probabilities for dust, clouds and clear sky to discriminate between them.

The dust model PDF directly provides information about dust particle size distribution (PSD) and mineralogical composition as well as a particle shape factor. The dust PSD is characterized by effective radius and mass-weighted mean diameter. The former is sensitive to the cross sectional area, whereas the latter represents the particle volume. PSD and mineralogical composition (also defining the particle shape model) are also used to dynamically calculate a conversion ratio for AOD from $10 \mu \mathrm{m}$ to $0.55 \mu \mathrm{m}$. Resulting optical depths (converted to $0.55 \mu \mathrm{m}$ based on retrieved mineralogy and particle size), the retrieved number of independent variables as well as dust particle size and mineralogical composition are depicted in fig. 2 for daytime overpasses of June 17, 2011 over the Sahara desert. 

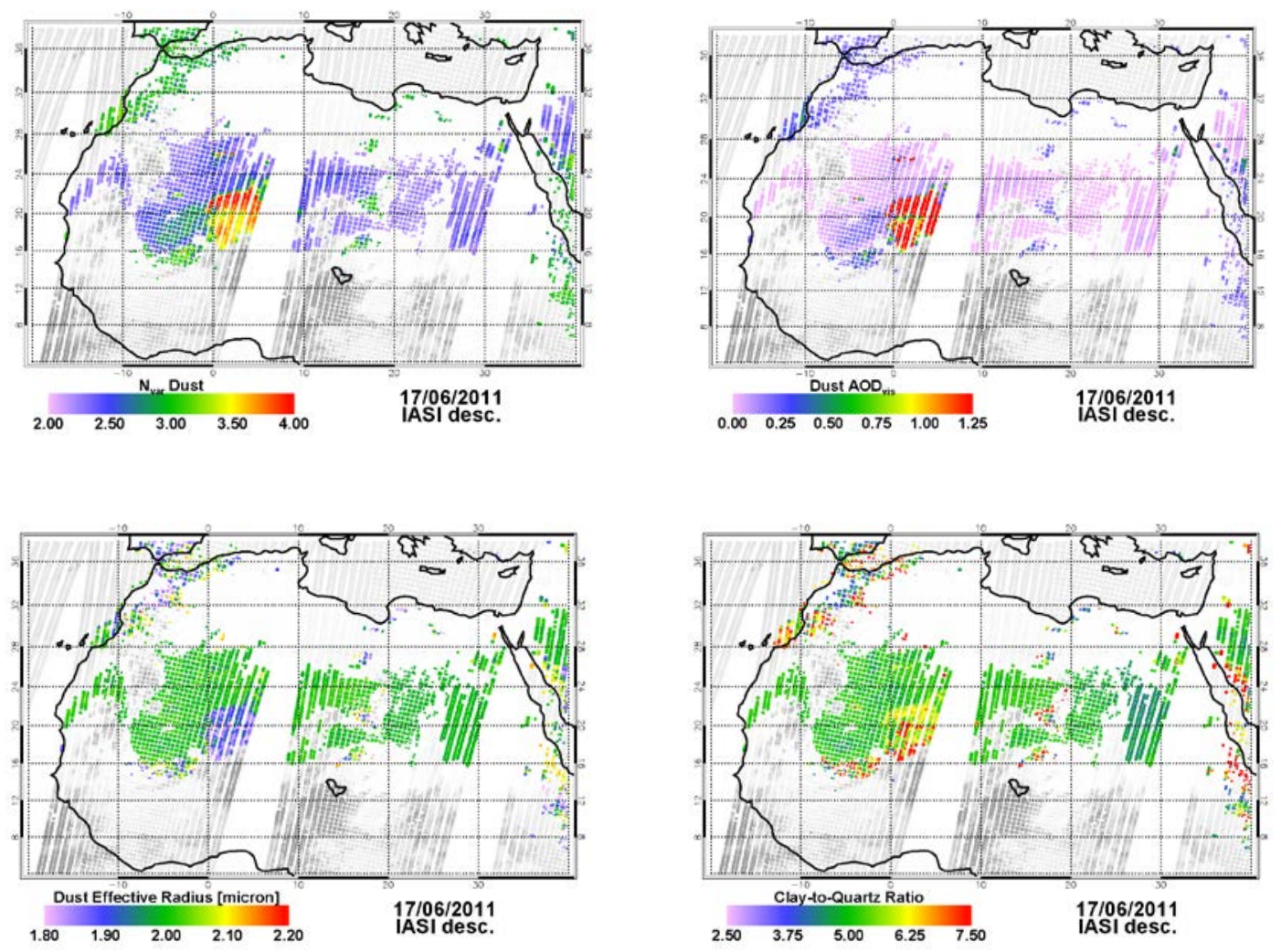

Fig. 2: Desert dust properties retrieved from space: number of independent signals (top left), dust optical depth (top right), dust particle size (bottom left) and mineralogical composition (bottom right) for daytime overpasses of June 17, 2011 over the Sahara desert.

\section{A climatology of dust properties: The IASI dataset within Aerosol_cci}

Within the Aerosol_cci project of the European Space Agency (ESA) IMARS dust observations with IASI starting in 2007 (start of the first Metop satellite) until present are produced and made publicly available. These include all above mentioned dust variables. This dataset is used to calculate a climatology of physico-chemical dust properties and to track significant dust events for identification of the corresponding dust sources.

\section{Conclusions}

The spectral shape of the desert dust absorption signal in the terrestrial infrared window region strongly depends on particle size, mineralogical composition and particle shape of the dust particles. Consequently methods including variability in these properties can be used to extract this information from hyperspectral satellite observations. The IMARS retrieval approach is used to create a climatology of desert dust properties from space.

\section{References:}

[1] L. Klüser, D., Martynenko, and T. Holzer-Popp, "Thermal infrared remote sensing of mineral dust over land and ocean: a spectral SVD based retrieval approach for IASI”, Atmos. Meas. Tech., 4, 757-773, doi:10.5194/amt-4-757-2011 (2011).

[2] L. Klüser, J.R., Banks, D., Martynenko, C., Bergemann, H.E., Brindley and T. Holzer-Popp, "Information content of space-borne hyperspectral infrared observations with respect to mineral dust properties”, Remote Sens. Environ., 156, 294-309, doi:0.1016/j.rse.2014.09.036 (2015).

[3] L. Klüser, C., Di Biagio, P.D., Kleiber, P., Formenti, and V.H. Grassian, "Optical properties of non-spherical desert dust particles in the terrestrial infrared - An asymptotic approximation approach”, J. Quant. Spec.

Radiative Trans., 178, 209-223, doi:10.1016/j.jqsrt.2015.11.020 (2016). 